\title{
DEVElopMEnt OF A Fully Automated TUNing System For ORgan PIPES
}

\author{
Clemens Sulz ${ }^{\mathrm{a}}$,Wendelin Eberle ${ }^{\mathrm{b}}$, Markus Trenker ${ }^{\mathrm{a}}$
}

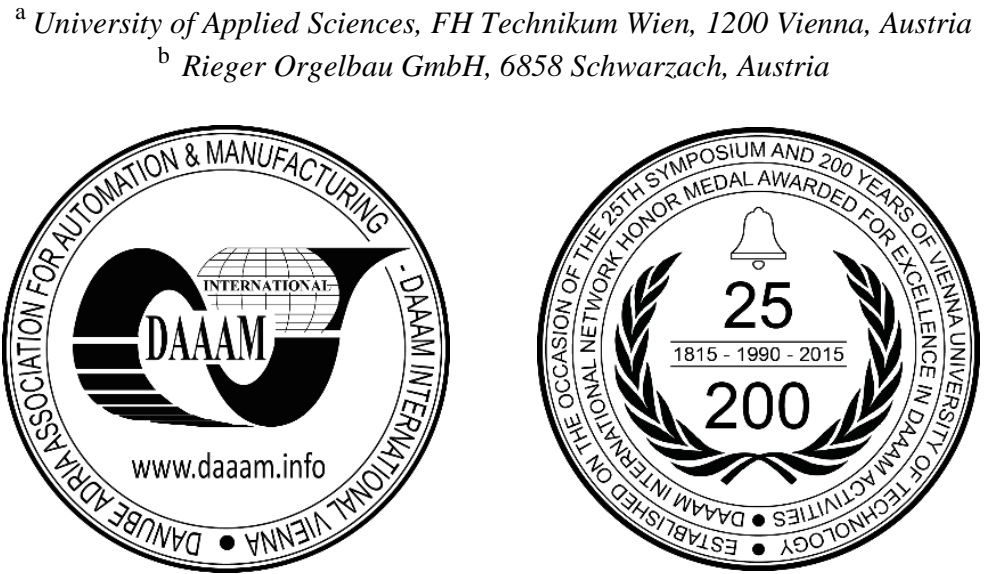

This Publication has to be referred as: Sulz, C[lemens]; Eberle, W[endelin] \& Trenker, M[arkus] (2016). Development of a Fully Automated Tuning System for Organ Pipes, Proceedings of the 27th DAAAM International Symposium, pp.0797-0803, B. Katalinic (Ed.), Published by DAAAM International, ISBN 978-3-902734-08-2, ISSN 1726-9679, Vienna, Austria

DOI: $10.2507 / 27$ th.daaam.proceedings. 115

\begin{abstract}
Many pipe organs consist of thousands of pipes, divided basically into two different types: flue pipes and reed pipes. Because of the fact, that the principle of sound generation differs, reed pipes must be tuned by hand periodically, which is a time-consuming and thus expensive process. The aim of this project was to do a feasibility study, to determine if this tuning process can be automated and to build up several prototypes for extensive testing. Thereby different actuator technologies were examined and evaluated. Finally a very cheap and compact actuator solution was developed. Appropriate software for controlling the system was programmed and the required drive electronics were developed. Tests with the prototypes have shown that the system is able to perform the tuning process in much shorter time than a human being with satisfying precision.
\end{abstract}

Keywords: organ, pipe; reed pipe; autonomous tuning system; automatic tuning application

\section{Introduction}

The pipe organ, called the king of instruments, has fascinated people for hundreds of years. It is the only instrument, which is played by feet and hands simultaneously, produces a huge range of tone colours and covers the whole frequency spectrum of the human hearing. Pressing a key causes air to stream into specific pipes, whereby each pipe produces one tone with a determined tone pitch and timbre. There can be thousands of pipes in a single pipe organ, with each pipe producing a unique sound. Basically, two types of pipes are used in pipe organs: flue pipes and reed pipes (left side of Fig. 1). The sound of the flue pipes is generated in the same way as in a real flute. The air stream strikes against the lip and begins oscillating. The result is a standing wave or vibrating column of air inside the pipe body. These are the facade pipes a beholder can generally see in a church and which represent the majority of the pipe stock. The pipes of the other type, reed pipes, work in a completely different way and are hidden inside the organ. Within the pipe foot there is a metal tongue, which begins to oscillate, if air flows through the pipe (right side of Fig. 1). The tuning spring is used to adjust the pitch of the reed pipe, because it defines the oscillatory length of the tongue. The tone colour of reed pipes allows imitating trumpets, clarinets or other wind instruments. 

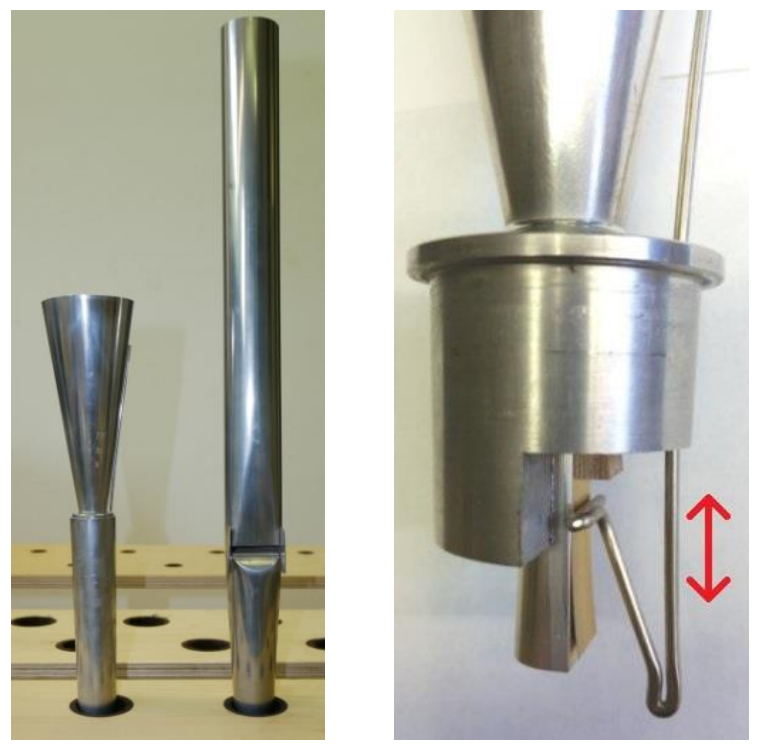

Fig. 1. Left picture: both types of organ pipes (reed pipe and flue pipe);

Right picture: inner parts of reed pipe (tuning spring is moved to tune the pipe)

\section{Problem description}

The pitch of flue pipes depends directly on the velocity of sound, which in turn depends on air temperature. So the pitch is lowered, if the temperature is decreased and vice versa. Because of the fact, that in reed pipes the tongue oscillates and not the air, the pitch of these pipes stays almost constant. A temperature change of just $1-2{ }^{\circ} \mathrm{C}$ causes an audible detuning of the organ. Not least because of the lower number of reed pipes and their easier tunability, the pitch of the reeds is tuned to the pitch of the flue pipes. To tune the pipes the tuning spring has to be moved up or down for each single pipe. Generally this tuning process requires two people (one sitting at the keyboard pressing down the keys) and takes between a few hours and several days for big organs. Because of the associated expense, the reed pipes often are not in tune and are not used by the organist. The aim of this project was to develop a system, which can tune reed pipes automatically. Refined, the aim was to determine whether a technical system is basically able to tune the reed pipes with satisfying precision in an acceptable amount of time. Furthermore, because of the number of reed pipes (usually a few hundred) the solution should be very cost-effective. Especially the little reed pipes were a challenging object of research due to the high sensibility of the tuning spring. Thereby movements of less than a micrometre are required to adjust the pitch exactly enough. The final stage of the project was to build a few prototypes to test and demonstrate the abilities of the system.

\section{State of the art}

At the beginning of this project an extensive market and patent review was done to find out, if any similar applications are already on the market. Thereby a few patents for automated organ tuning were found belonging to the German organ builder Voigt ([1], [2] and [3]). Furthermore, a project within the framework of a bachelor thesis by Fachhochschule Kiel [4] was found. But in contrast to the idea of automating the tuning process for reed pipes all these applications are developed to modify the pitch of flue pipes, whereby these projects are primarily concerned with conceptual studies. The company Rieger Orgelbau [5], which was the main cooperation partner for this project, has developed a system, which allows the tuning person to control the organ with a smartphone app. Specifically it is possible to play the keys of the organ via the smartphone, so the second person is no longer needed. This system represented the newest state of technology at the beginning of this project. If an actuator, which should be developed in the course of this project, would be combined with this system, a fully automated tuning application would be established.

\section{Actuator research}

Following the analysis of the mentioned system a research on actuators was performed. Thereby the most important criteria were cost efficiency and space requirements. Furthermore the components and structure of reed pipes should not be modified, or if it is unavoidable, as little as possible. This would make it feasible to upgrade already existing organs with the tuning system. Before the research took place, force investigations on various tuning springs on three different pipes of different size were conducted to determine, how much force an actuator should be able to apply. The highest value which was measured was $6,0 \mathrm{~N}$. Including an appropriate safety surcharge for the following research a minimum guide value of $10 \mathrm{~N}$ was defined. 


\subsection{Piezoelectric drives}

Because of the required precision, piezoelectric drives were examined as a first step. One possible new type of piezo drive is the motor X15G (Fig. 2) from Elliptec [6]. If the piezo crystal inside this actuator is driven by the natural resonant frequency of the whole actuator, the rotor begins to move forward. With a second specific frequency the motor could also be moved backwards. According to the datasheet the drive can also be used as a linear actuator, whereby the drive could be attached directly to the tuning spring to move it up or down (see right side of Fig. 2). Unfortunately, it became apparent that this drive can only raise $1.2 \mathrm{~N}$, which is much too little for this application.
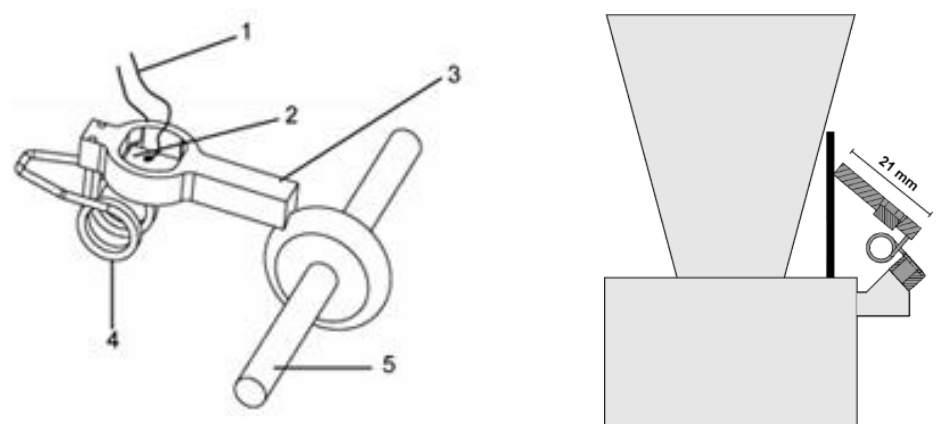

Fig. 2. Piezo motor Elliptec X15G; Left side: 1...wires, 2...piezo ceramic, 3...resonator, 4...spring, 5...rotor

(Source: based on [6]); Right side: possible implementation on reed pipe

A second interesting piezo drive is the so-called Squiggle motor (Fig. 3) from New Scale Technologies [7]. Because of the coordinated movement of four piezo elements, the threaded rod inside the drive begins to move translationally without twist. If the tuning spring would be threaded, the Squiggle motor could be attached to it directly. In this case this drive would be very interesting (very low space requirement), but it also became obvious that the force of the motor is too low (max. $0.2 \mathrm{~N}$ ) for the tuning application.

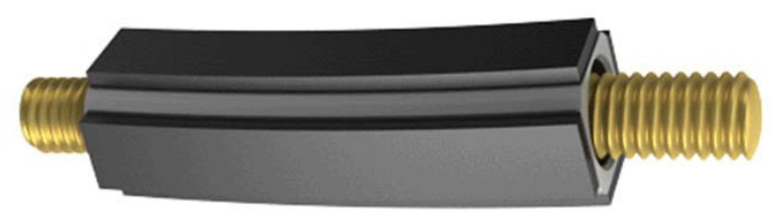

Fig. 3. Squiggle motor (Source: [7])

A last piezoelectric drive, which was investigated, is the Piezomike (Fig. 4) from PI GmbH [8]. With 20N thrust, it was the first piezo device found, which would be strong enough for the tuning application. The piezo crystal inside the actuator is expanded slowly because of the controlled increase of voltage, whereby the gripper starts rotating the screw. If the final position is reached, the voltage is switched off and the gripper goes back to the starting position jerkily without moving the screw. Right side of Fig. 4 shows a schematic diagram for a possible implementation on a reed pipe with a spring, whereby the tuning spring is pulled against the Piezomike. A resulting advantage with this kind of drive would be the possibility to tune the pipe manually through rotation of the screw shaft without disassembling the tuning system. Due to the good applicability the manufacturer of this piezo drive was contacted to get the price of the system. The exorbitantly high price of about $500 \$ /$ pcs. inhibits the application in this project.
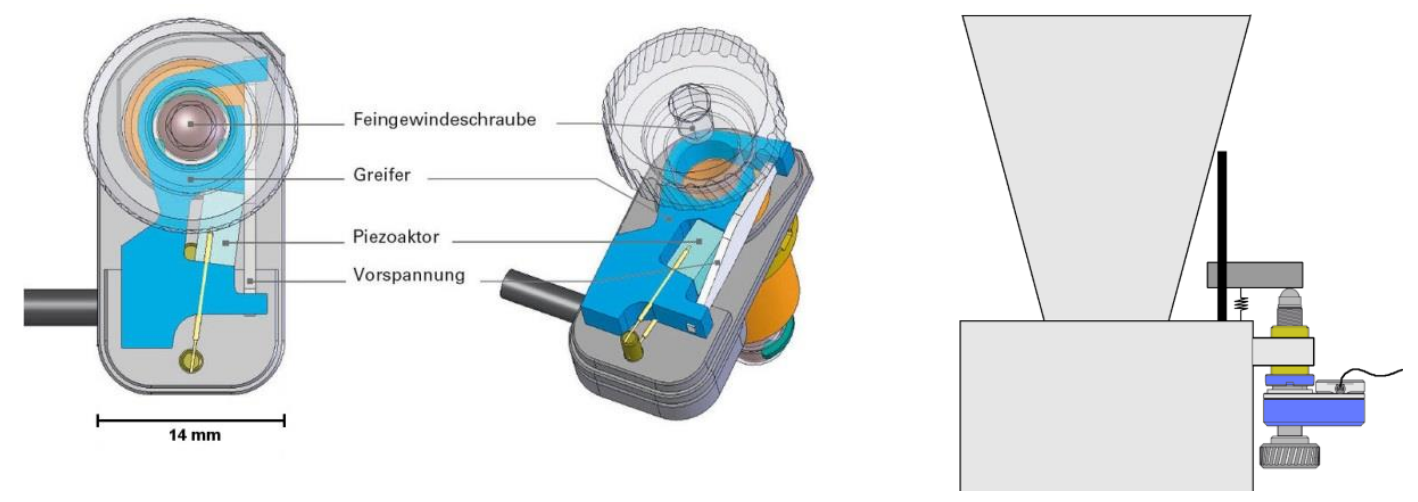

Fig. 4. Piezomike and possible implementation (Source: [8]) 


\subsection{Stepper motors}

Because of the possibility of fine positioning of stepper motors, these drives were investigated following the piezo drives. Thereby stepper motors with already mounted threaded-spindle shafts were examined in detail. A possible application is illustrated in Fig. 5.

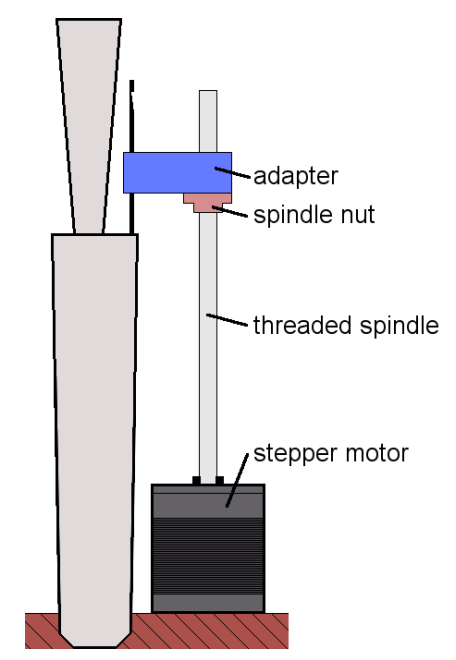

Fig. 5. Stepper motor with threaded shaft as tuning device

The spindle nut in combination with a connected adapter part could transform the rotation into translational movements for the tuning spring. This would certainly be a solution, which would generate the required forces. But because of the centric motor shaft and the frame size of a stepper motor this motor type would not fit between the pipes.

\subsection{DC gearbox drives}

As a smaller and cheaper alternative to stepper motors DC gearbox motors were explored. Thereby the structure with a threaded shaft, as in the last section on stepper motors, should be used. Due to the gear reduction such a motor could be significantly smaller. Also the drive electronics would be simpler to implement.

\section{Practical realization}

In the following section the implemented solution is described in detail.

\subsection{Implemented drive technology}

Owing to the fact, that the last described drive technology with a gearbox motor seems to be the best one, it was chosen to build a prototype and for further evaluation. An appropriate gearbox motor was available, wherefore this drive was used for a first prototype (Fig. 6).

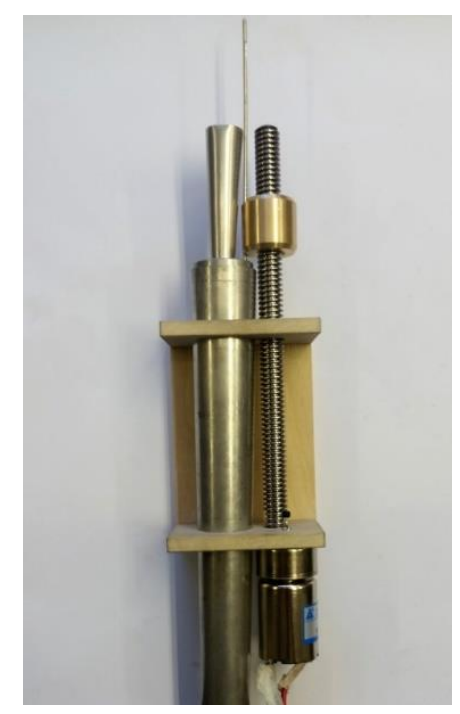

Fig. 6. First prototype with gearbox motor 
For testing the prototype, software, which will be described in section 5.3, was developed in parallel. With the experimental setup, first successes in tuning the pipe were achieved. Nonetheless searching for alternative gearbox drives was continued, whereby a very compact and cheap gearbox motor was found, which is perfectly suited to the tuning application. This drive already has a threaded M3 or M4 (selectable) output flange. Furthermore, there is an alternative "'Flip-Type"' of this motor available. Thereby the input and output shaft are on the same side of the gearbox. This allows a more compact design and the reduction of the distance between the tuning spring and the threaded shaft. In low quantities this version is not available with a threaded shaft, but according to the manufacturer it could be modified for higher order quantities. For prototyping, an adapter for mounting a threaded rod was manufactured manually. In Fig. 7 prototypes with both kinds of motors are pictured. Note that the left prototype contains the same pipe as in Fig. 6 to enable one to see the difference in size.
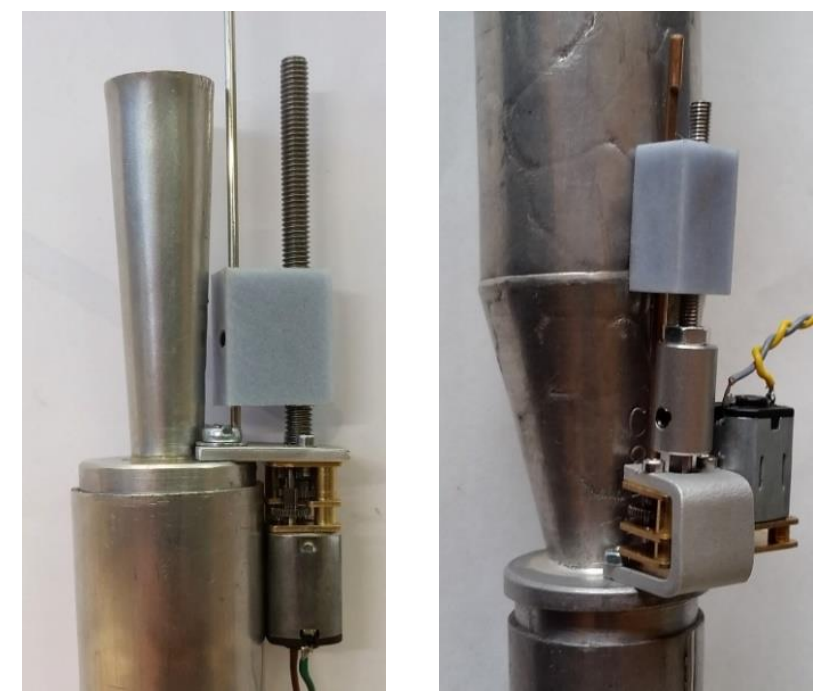

Fig. 7. Prototypes with compact gearbox motors (motor housing length with gearbox is $25 \mathrm{~mm}$ )

To transform the rotating movement of the spindle into translation for the tuning spring, an adapter component of high-strength plastic was manufactured. In this component the tuning spring is fixed with a grub screw. If this single screw is loosened, the pipe can be tuned manually without disassembling the automatic tuning system. Thus an excellent, mechanical solution for the tuning system was found. Because of the low thread pitch, high precision positioning in micrometre range creates no problems for this actuator system. Additionally, the low thread pitch and the high gear reduction result in the very low drive torque needed for the motor. Performed force measurements showed, that the solution can generate about $60 \mathrm{~N}$ before the drive blocks, which is 10 -times more than required.

To move the tuning spring and correct the pitch of the pipes, a control loop is necessary. This loop must contain the final control element or actuator, which executes the calculated move, frequency detection and a logic unit or software, which processes the frequency measurements. In Figure 8 such a control loop is depicted.

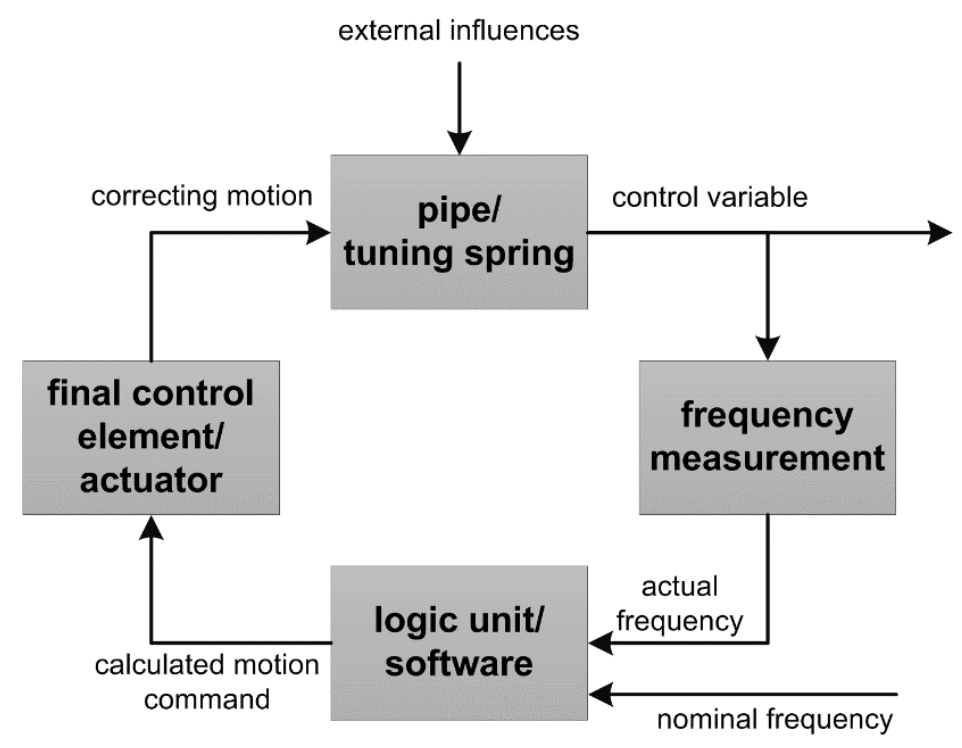

Fig.8. Control circuit for automated pipe tuning 


\subsection{Frequency detection}

At the beginning of this project the bought-in tuning device TLA CTS-32-C [9] was used for pitch detection. It communicates with the software part over an USB-Interface and was especially developed for organ builders and their needs. Because of the high price of the tuning device, an own solution for detecting the frequency was developed. Using a variable bandpass filter, it is possible to extract a sinusoidal wave with the fundamental frequency of the pipe from a complex audio signal, which is recorded by a microphone. Through detecting the zero-crossing-rate of this sinus the pitch of the pipe can be calculated directly, using an Arduino platform for this purpose in prototype stage.

\subsection{Software}

For calculating the required movements of the motors from the frequency measurement, appropriate software was developed in C\#. For controlling the tuning system in the prototyping phase a graphical user interface (GUI) was designed. The tuning device and the electronics (described in the following section) are connected via USB to the computer, on which the software is executed. On the GUI the current divergence to nominal frequency is charted in real-time. The motors are not driven continuously, but stepwise. The length of the switched-on pulses depends on the divergence to nominal frequency of the pipe, followed by a stop until the next pulse length is calculated. This stepwise mode is needed because of the very high sensibility of the reed. At the smallest pipes 1 micrometre motion of the tuning spring results in 0.5 cents deviation of pitch.

\subsection{Electronics}

To transform the calculated pulses from the software into voltage for the motors, drive electronics and an appropriate logic unit are needed. Therefore, an Arduino board with three motor shields (extension boards) was used. Each board can drive two motors, so six pipes can be connected simultaneously for prototyping. Furthermore, the motor shields support motor current measurement, so it can be detected without additional sensors, if the motor is stalling, e.g. if the tuning spring has reached its end position.

\section{Results}

After finishing the constructing phase, the prototyping setup was tested extensively. An endurance test was performed with one pipe to verify fatigue strength of the system. Thereby the motor moved the tuning spring for about 20 hours continuously (3715 tuning cycles), until one gear wheel was abraded. This number of tuning cycles would never be reached in a real organ, so the drive is applicable from this point of view.

The precision and the speed of the automated tuning process meet the requirements set for this project. A pipe can be tuned in less than ten seconds with satisfying precision $( \pm 0.5$ cents), whereby the manual process takes about 30 seconds for each pipe. The system can perform tuning even more accurately, whereby the tuning time increases.

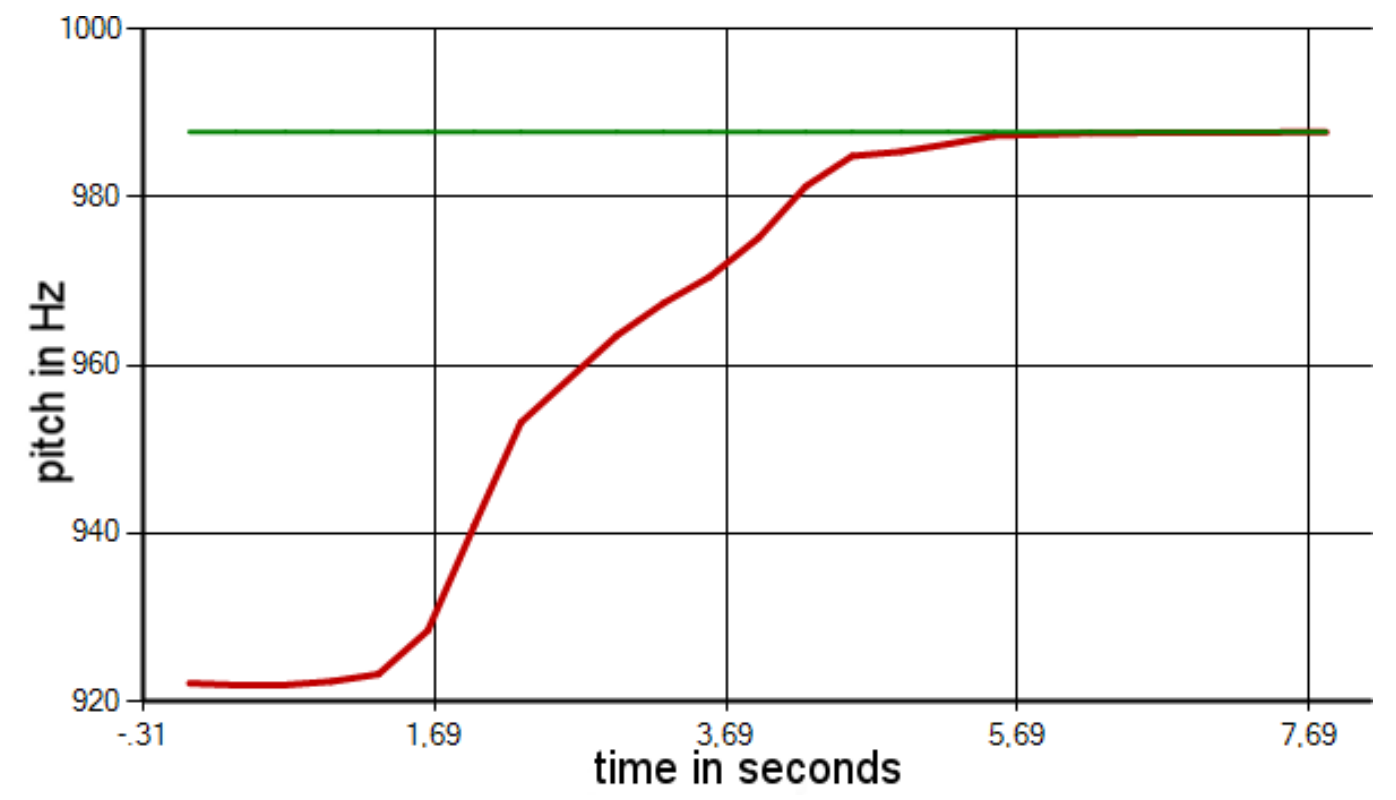

Fig. 9. Resulting tuning process of reed pipe (green...nominal value, red...actual value) 


\section{Conclusion and outlook}

Overall, the main aim of this project, to evaluate the possibility of automated reed pipe tuning, was reached at an early stage and extensive additional developmental work was done. Because of the low price and the small size of the implemented actuator the results actually exceeded the author's own expectations by far. In future work the software should be transformed from PC to an embedded system and should be integrated into the real organ control system. Thereby the organ could be programmed to tune itself at specific dates or tuned by starting the process from a smartphone from anywhere. Using more than one bandpass filter would enable tuning several pipes simultaneously. That would be a significant advantage over to manual pipe tuning.

\section{References}

[1] Voigt, D. \& Voigt, M. (2012). Pfeifenorgel mit selbstregulierender Stimmung, Patent DE102011013444

[2] Voigt, M. (2014). Stimmungseinrichtung für Orgelpfeifen, Patent DE102012021644

[3] Voigt, M. (2015). Stimmungseinrichtung für gedackte Orgelpfeifen, Patent DE102013012821

[4] Bothe, T. \& Kablitz, J. (2014). Selbststimmende Orgelpfeife, Bachelorprojekt, FH Kiel

[5] Rieger Orgelbau GmbH (2016). Rieger Orgelbau, http://www.rieger-orgelbau.at/ Accessed: 2016-10-01

[6] Elliptec GmbH (2016). Elliptec Motor X15G, Available at: http://www.elliptec.com/de/produkte/motor-x15g/, Accessed 2016-10-01

[7] New Scale Technologies (2016). Squiggle micro motor technology, Available at: http://www.newscaletech.com/technology/squiggle-motors.php, Accessed 2016-10-01

[8] Physik Instrumente (PI) GmbH (2016). Piezomike Linearaktoren, Available at: http://www.physikinstrumente.de/technologie/piezomike-linearmotoren.html, Accessed 2016-10-01

[9] Langbein, T. (2008). Tuning Set CTS-32-C,Bedienungsanleitung, Abtsgmünd, Available at: http://www.tuningset.de/Beda32C_D_Internet.pdf, Accessed 2016-10-01 